\title{
Application of Ray Model for Terahertz Transmission Simulations of Tube Waveguide
}

\author{
Yi Zhong1, Xuehui Lu², Guoxing Xie', Chengbin Jing ${ }^{1,2 *}$ \\ ${ }^{1}$ Department of Materials, School of Physics and Materials Science, East China Normal University, Shanghai, China \\ ${ }^{2}$ Key Laboratory of Polar Materials and Devices, Ministry of Education, School of Information Science Technology, East China \\ Normal University, Shanghai, China \\ Email: *cbjing@ee.ecnu.edu.cn
}

How to cite this paper: Zhong, Y., Lu, X.H., Xie, G.X. and Jing, C.B. (2018) Application of Ray Model for Terahertz Transmission Simulations of Tube Waveguide. Journal of Computer and Communications, 6, 143-147.

https://doi.org/10.4236/jcc.2018.612014

Received: December 18, 2018

Accepted: December 23, 2018

Published: December 26, 2018

\begin{abstract}
Terahertz $(\mathrm{THz})$ transmission simulations play an important role in $\mathrm{THz}$ technology researches, especially for the structural design of a $\mathrm{THz}$ waveguide. Ray model takes into account both structure parameter of waveguide and the divergence angle of beam light and could be an alternative way for $\mathrm{THz}$ transmission behavior simulations. In this paper, the ray model is used to calculate the transmission loss of tube waveguide, and the simulated transmission losses are presented to compare with the results calculated by COMSOL. The suitable $\mathrm{THz}$ frequency range of ray model is discussed by analyzing the transmission loss spectra of tube waveguides with various core sizes. The credibility of ray model on terahertz transmission simulations is demonstrated based on the experimental results tested by THz-TDS and calculated results.
\end{abstract}

\section{Keywords}

THz Waveguide, Transmission Loss, Ray Model

\section{Introduction}

In recent years, terahertz $(\mathrm{THz})$ waveguides and fibers have been extensively investigated. The dry air is demonstrated to be the most transparent medium in the $\mathrm{THz}$ region and can be used for $\mathrm{THz}$ transmission. Many types of $\mathrm{THz}$ waveguides are designed to transmit $\mathrm{THz}$ radiations with dry air medium, including photonic crystal fibers [1], metal/dielectric hollow glass waveguides [2] [3], and polymer tube waveguides [4] [5] [6]. The polymer tube waveguide, which consists of an air core and a dielectric layer, is considered as a good candidate for the transmission of $\mathrm{THz}$ radiations. It exhibits low transmission attenuation, 
because $\mathrm{THz}$ waves transmit in the air core of the tube waveguide. And the structure of tube waveguide is simple and easy for fabrication. Figure 1 shows the transverse cross-section of the tube waveguide. It has an air-core (refractive index $\mathrm{n}_{1}$ ) with inner diameter $\mathrm{D}$ and a thin cladding (refractive index $\mathrm{n}_{2}$ ) with thickness t.

Before performing the fabrication procedure of a tube waveguide, the structure of the waveguide needs to be carefully designed to obtain a low transmission loss in theory. The simulation software COMSOL Multiphysics (hereafter referred to as COMSOL), which based on Finite Element Method (FEM), is widely used to the theoretical analysis of the $\mathrm{THz}$ transmission. The simulation needs the parameter of $\mathrm{D}, \mathrm{t}, \mathrm{n}_{1}$, and $\mathrm{n}_{2}$. And the parameter of transmission, including modal distribution, transmission loss in different modes, effective refractive index, and group velocity dispersion, can be obtained with the COMSOL. However, in the practical $\mathrm{THz}$ transmission, the transmission loss depends on not only the structure but also the divergence angle of $\mathrm{THz}$ beam. And the transmission loss is a combination of transmission losses in multiple modes when the $\mathrm{THz}$ radiation is multi-mode transmission.

The ray model [7], which is based on geometric optics method, can be used to calculate the transmission loss for tube waveguides and metal/dielectric hollow glass waveguides when the inner diameter of waveguide is much larger than the transmission wavelength. The transmission loss calculated by ray model is connected with the structure of the waveguide and the divergence angle of $\mathrm{THz}$ beam and takes no account of the transmission modes. And the calculation speed of ray model is much faster than COMSOL. Hence the ray model could be an alternative way for $\mathrm{THz}$ transmission behavior simulations. The ray model has been used to calculate transmission loss in metal/dielectric hollow glass waveguide for delivery of mid- and far-infrared $(2.5 \mathrm{THz})$ radiations [2]. But whether ray model is applicable or not in lower frequency (larger wavelength)? In this paper, we demonstrated ray model can be used to calculate transmission loss for tube waveguides, even at the frequency as low as $0.5 \mathrm{THz}$.

\section{Comparison of COMSOL and Ray Model}

The transmission loss spectra are calculated by ray model and COMSOL, respectively. The $\mathrm{D}, \mathrm{t}, \mathrm{n}_{1}$, and $\mathrm{n}_{2}$ are $4 \mathrm{~mm}, 0.2 \mathrm{~mm}, 1$, and 1.4. And the divergence angle of $\mathrm{THz}$ beam is $6^{\circ}$ for ray model. As can be seen in Figure 2, the transmission

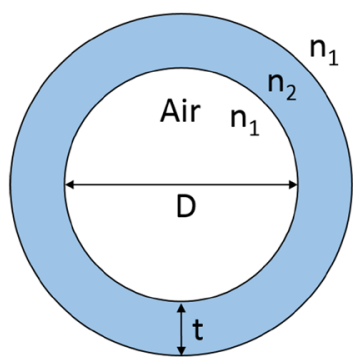

Figure 1. Transverse cross-section of the tube waveguide. 




Figure 2. Comparison of transmission loss calculated from ray model and COMSOL.

loss curve calculated by ray model is the black line, and there are four transmission loss curves calculated by COMSOL depending on the four lowest modes $\left(\mathrm{HE}_{11}, \mathrm{HE}_{21}, \mathrm{TM}_{01}\right.$, and $\left.\mathrm{TE}_{01}\right)$. In the $0.8-2 \mathrm{THz}$, the transmission losses calculated by ray model and COMSOL are approximated. But when the frequency is lower than $0.65 \mathrm{THz}$, the transmission loss curves calculated by two methods are divergent. Because the transmission wavelength is close to the inner diameter of waveguide, and the ray model is no longer applicable. Thus the applicable frequency range of the ray model has a lower limit (called limit frequency) for a certain inner diameter of waveguide.

\section{Exploration of Limit Frequency}

To research the relationship between the limit frequency of ray model and the inner diameter of waveguide, the transmission loss spectra from four tube waveguides, with different inner diameter, are calculated. The D is $2 \mathrm{~mm}, 4 \mathrm{~mm}, 6$ $\mathrm{mm}$, and $8 \mathrm{~mm}$, respectively, and other parameters are not change. And for convenience, only the fundamental model $\left(\mathrm{HE}_{11}\right)$ is given in the calculation result of COMSOL. In Figure 3, the dash line gives the limit frequency, which is 1 $\mathrm{THz}, 0.65 \mathrm{THz}, 0.55 \mathrm{THz}$, and $0.45 \mathrm{THz}$ for the inner diameter $2 \mathrm{~mm}, 4 \mathrm{~mm}, 6$ $\mathrm{mm}$, and $8 \mathrm{~mm}$. It shows that the limit frequency decreases as the inner diameter of waveguides increases.

In order to clearly indicate the relationship between the limit transmission wavelength and the inner diameter. The limit frequency is translated into limit transmission wavelength. As can be seen in Figure 4, the relationship between the limit transmission wavelength and the inner diameter is approximately $\lambda=$ 0.092D.

\section{Comparison with Experimental Result}

To prove the applicability of ray model, a Teflon (refractive index 1.4) tube is measured by $\mathrm{THz}$ time-domain spectroscopy (THz-TDS) system. The practical $\mathrm{D}, \mathrm{t}, \mathrm{n}_{1}$, and $\mathrm{n}_{2}$ are $3.4 \mathrm{~mm}, 0.16 \mathrm{~mm}, 1$, and 1.4. And the practical divergence angle of $\mathrm{THz}$ beam is $36^{\circ}$. Figure 5 gives the experimental results from THz-TDS 

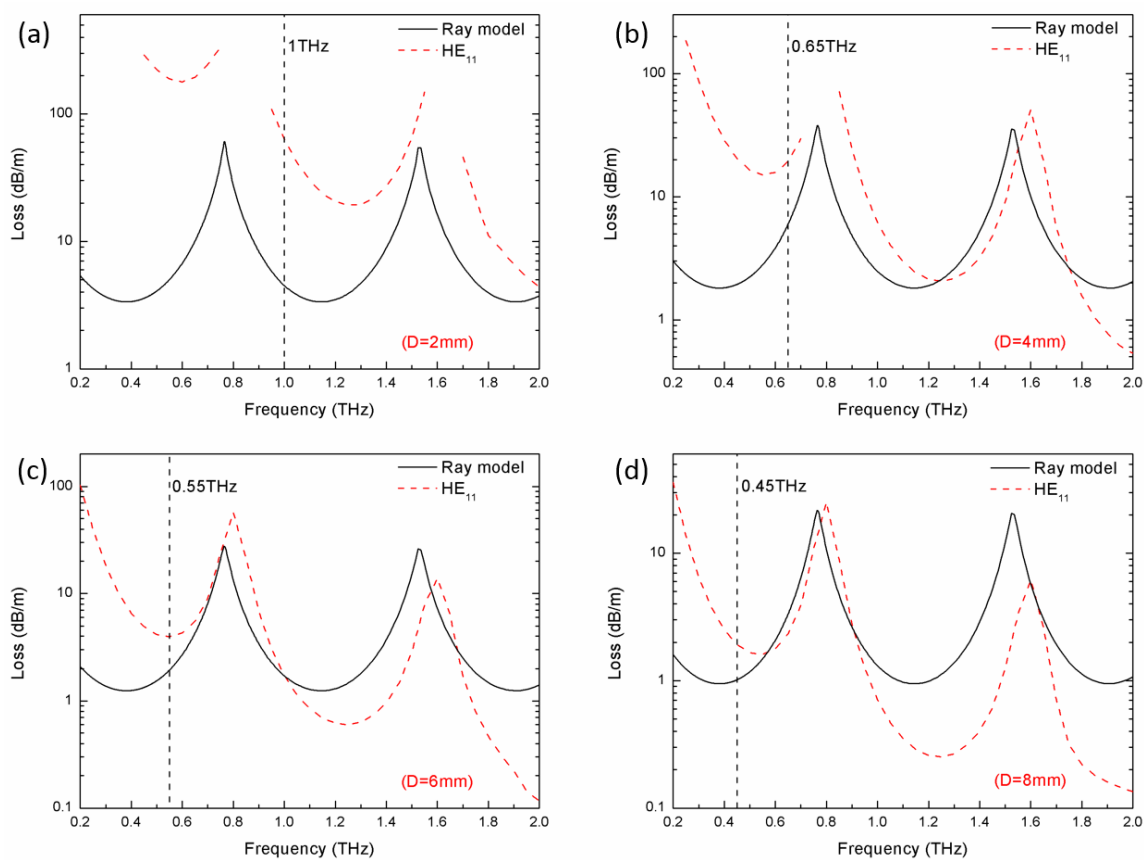

Figure 3. Comparison of transmission loss from four inner diameters waveguide. The corresponding inner diameter of (a), (b), (c), and (d) is $2 \mathrm{~mm}, 4 \mathrm{~mm}, 6 \mathrm{~mm}$, and $8 \mathrm{~mm}$.

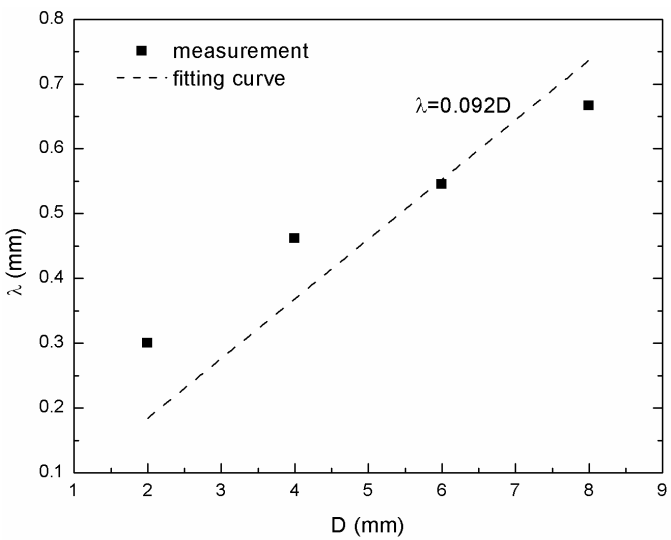

Figure 4. Relationship between the limit transmission wavelength $(\lambda)$ and the inner diameter (D).

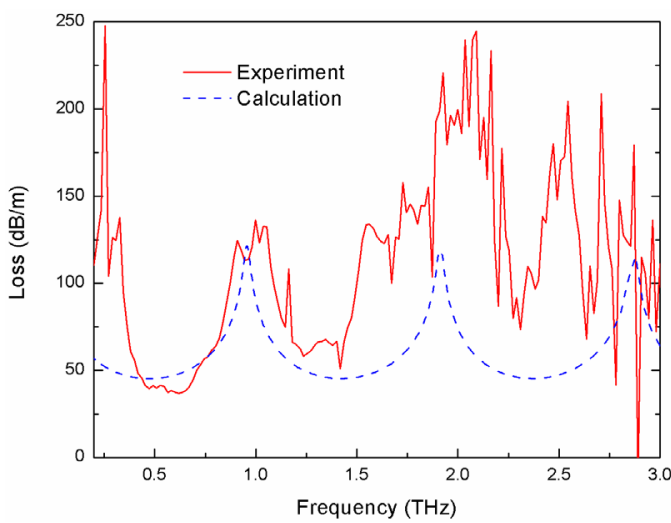

Figure 5. Experimental and calculated results of Teflon tube waveguide. 
and the calculation from ray model. The curves between experiment and calculation are approximately consistent in $0.4-3 \mathrm{THz}$.

\section{Conclusion}

In this paper we use ray model to calculation the transmission loss of tube waveguide, and the transmission loss curves are presented to compare with the results calculated by COMSOL. In a range of region, the ray model is an alternative way for the calculation of $\mathrm{THz}$ transmission loss. Moreover, the relationship between limit transmission wavelength and inner diameter of waveguide is determined to $\lambda=0.092 \mathrm{D}$. The experiments testify that the result calculated by ray model is credible.

\section{Acknowledgements}

The authors acknowledge the financial support provided within National Natural Science Foundation of China (NSFC) (61775060, 61275100, and 61761136006).

\section{Conflicts of Interest}

The authors declare no conflicts of interest regarding the publication of this paper.

\section{References}

[1] Yang, J., Zhao, J., Gong, C., Tian, H., Sun, L., Chen, P. and Liu, W. (2016) 3D Printed Low-Loss $\mathrm{THz}$ Waveguide Based on Kagome Photonic Crystal Structure. Optics Express, 24, 22454-22460. https://doi.org/10.1364/OE.24.022454

[2] Bowden, B., Harrington, J.A. and Mitrofanov, O. (2007) Silver/Polystyrene-Coated Hollow Glass Waveguides for the Transmission of Terahertz Radiation. Optics Letters, 32, 2945-2947. https://doi.org/10.1364/OL.32.002945

[3] Navarro-Cía, M., Melzer, J.E., Harrington, J.A. and Mitrofanov, O. (2015) Silver-Coated Teflon Tubes for Waveguiding at $1-2 \mathrm{THz}$. Journal of Infrared, Millimeter, and Terahertz Waves, 36, 542-555. https://doi.org/10.1007/s10762-015-0157-5

[4] Lai, C.H., Hsueh, Y.C., Chen, H.W., Huang, Y.J., Chang, H.C. and Sun, C.K. (2009) Low-Index Terahertz Pipe Waveguides. Optics Letters, 34, 3457-3459. https://doi.org/10.1364/OL.34.003457

[5] Bao, H., Nielsen, K., Bang, O. and Jepsen, P.U. (2015) Dielectric Tube Waveguides with Absorptive Cladding for Broadband, Low-Dispersion and Low Loss $\mathrm{THz}$ Guiding. Scientific Reports, 5, 7620. https://doi.org/10.1038/srep07620

[6] Lai, C.H., Yeh, Y.S., Yeh, C.A. and Wang, Y.K. (2018) Effective Bandwidth of Terahertz Antiresonant Reflecting Pipe Waveguide. Optics Express, 26, 6456-6465. https://doi.org/10.1364/OE.26.006456

[7] Miyagi, M. (1985) Waveguide-Loss Evaluation in Circular Hollow Waveguides and Its Ray-Optical Treatment. Journal of Lightwave Technology, 3, 303-307. https://doi.org/10.1109/JLT.1985.1074177 